\title{
ESTRATEGIA ORGANIZACIONAL Y LA RENTABILIDAD EN EMPRESAS DEL SECTOR AUTOMOTRIZ DE LA ZONA CENTRAL DEL ECUADOR.
}

\section{STRATEGY AND ORGANIZATIONAL PERFORMANCE IN AUTOMOTIVE COMPANIES OF ECUADOR CENTRAL AREA SECTOR.}

\section{Recebimento: 8/9/2016- Aceite: 11/11/2016- Publicação: 29/11/2016 Processo de Avaliação: Double Blind Review}

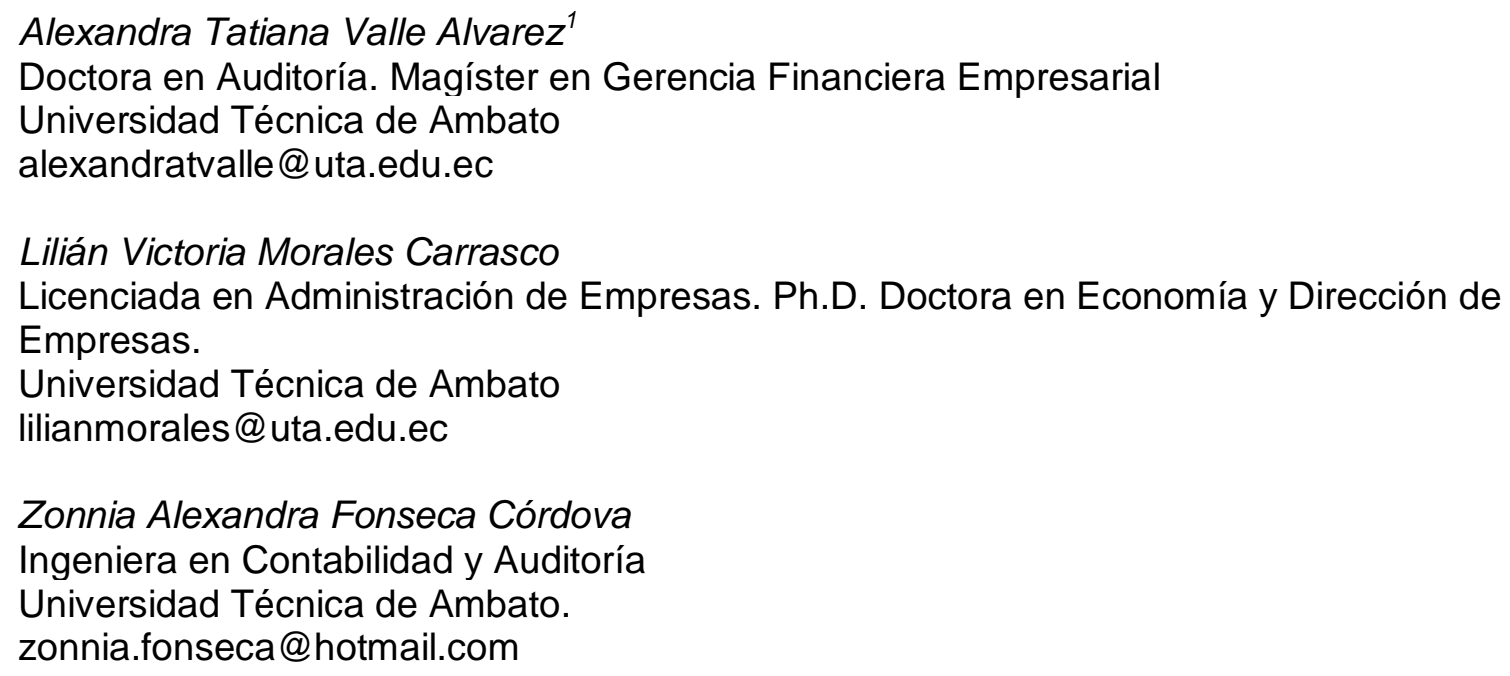

\section{RESUMEN}

El objetivo de esta investigación es aproximarnos al estudio de la estrategia organizacional y la rentabilidad en las empresas representativas del sector automotriz de la Zona Central del Ecuador durante los años 2013 al 2015. En base a la tipología genérica propuesta por Miles y Snow en torno a la estrategia y el estudio de la rentabilidad, medida a través del ROI y el EBITDA, se evaluaron cualitativa y cuantitativamente a siete empresas del sector, identificándose inicialmente características en torno a cinco factores: mercado, eficiencia, innovación y estructura, en base a los cuales se diferenciaron arquetipos estratégicos en las empresas que las identifican como: Prospectiva, Analizadora o Defensiva. No se hicieron evidentes casos de empresas Reactivas en la población estudiada. Los principales resultados

\footnotetext{
${ }^{1}$ Autor para correspondencia: Universidad Técnica de Ambato: Av. de los Chasquis y Río Cutuchi. Ciudadela Universitaria. Ambato-Ecuador.alexandratvalle@uta.edu.ec
} 
de la investigación muestran a través del análisis descriptivo que la caracterización de las empresa Prospectiva y Analizadora identificada en la mayoría de empresas estudiadas está ligada a mayores niveles de rentabilidad, incluso cercanos a los reportados por la industria, que aquella que habría aplicado una estrategia de carácter Defensiva, con desempeño comparativamente menor en ROI y EBITDA.

Palabras clave: estrategia, rentabilidad, ROI, EBITDA.

\section{ABSTRACT}

The purpose of this research is to approach the study of organizational strategy and the profitability in the companies of the companies that represent the automotive sector of the Central Zone of Ecuador for the years 2013 to 2015. Based on the generic typology proposed by Miles and Snow regarding the strategy and the study of profitability, measured by ROI and EBITDA, seven companies have been qualitatively and quantitatively evaluated, identifying initially characteristics around five factors: market, efficiency, innovation and structure, which were the base to differentiate strategic archetypes in the companies, that identify them as: Prospective, Analyzer or Defensive. There was no evidence of obvious cases of Reactive companies in the studied population. The main results of the research show through descriptive analysis that the characterization of the companies Prospective and Analyzer, identified in most of the studied companies, is linked to higher levels of profitability, even close to those reported by the industry, than in those that have applied a Defensive character strategy, with comparatively lower ROI and EBITDA performance.

Keywords: strategy, profitability, ROI, EBITDA

\section{INTRODUCCIÓN}

El actual contexto socioeconómico en el que se ha desarrollado la actividad empresarial a nivel global, plantea cada vez nuevas presiones en formas de oportunidades y amenazas que son evaluadas por los responsables de la gestión en las organizaciones, las 
decisiones o caminos escogidos son identificados como aquellas estrategias diferenciadoras que les permitirá alcanzar sus objetivos a largo plazo. La estrategia por tanto, se considera clave para el logro de ventajas competitivas sostenibles, por cuanto articula la forma en que la organización encara sus fortalezas y debilidades, y configura sus actividades de mercado para ganar dicha ventaja. (Sheppeck \& Militello, 2000).

El sector automotriz tiene una participación importante en la economía ecuatoriana, debido a los ingresos que genera en todas las actividades económicas directas e indirectas que involucra, además de su impacto en la generación de empleo en las diferentes partes de su cadena, desde el ensamblaje hasta la distribución y ventas. Esta industria ha impulsado a otras del sector productivo como la siderúrgica, metalúrgica, metalmecánica, minera, petrolera, petroquímica, del plástico, vidrio, electricidad e informática, industrias claves en la fabricación de los vehículos. De este modo, el sector automotriz integra a diferentes actores, tanto para las firmas autopartistas proveedoras de partes y piezas; así como para las ensambladoras que son las firmas que imponen los estándares productivos de la cadena.

Según la Asociación de Concesionarios Automotrices del Ecuador (2014), desde el año 2000, luego del proceso de dolarización en el Ecuador, la ventas de vehículos en el Ecuador mostraron un crecimiento importante y sostenido durante la siguiente década, sin embargo, a partir del año 2012 se produjo un decremento del 13\% y del 6\% del año 2011 al 2012 y del 2012 al 2013 respectivamente, esto es una disminución de 139.893 unidades en el 2011 a 121.446 en el año 2012 y a 113.812 para el 2013. Para el año 2014 se produjo un incremento del 5,5\%, pasando a 120.060 unidades vendidas en la industria automotriz.

El comportamiento del sector automotriz durante los últimos años se ha visto impactado por el entorno normativo, con las medidas que ha adoptado el Gobierno Nacional y que han significado la imposición de restricciones a las importaciones de vehículos y el aumento de aranceles para este sector. Inicialmente el Gobierno Nacional implementó un régimen de licencias para controlar las importaciones de vehículos armados, debido al déficit importante de la balanza comercial en el año 2010, que según datos del Banco Central del Ecuador ascendió a USD1.978,9 millones.

Con la entrada en vigencia de la medida oficializada el 15 de junio de 2012, con Resolución COMEX No.66, se instrumentó el tercer cambio gubernamental en nueve meses, tendiente a restringir las importaciones de vehículos. Para la asignación de las cuotas o cupos de importación impuestos mediante la "restricción ambiental", se consideraron los montos totales en unidades y dólares FOB importados por las empresas del sector en ese año 2010 con una reducción del 30\%. Los cupos asignados para cada importador serían los mismos para los años 2012-2013 y 2014. Adicionalmente, con la Ley de Regulación de Créditos de Vivienda y Vehículos (Junio de 2012) el mercado automotriz experimentó un incremento generalizado de precios en los segmentos restringidos (automóviles, SUV's, camionetas y mini VAN's) lo cual limitó la oferta de varios modelos y se dificultó el acceso por parte de los usuarios a opciones de financiamiento para la adquisición de un vehículo. 
La oferta nacional en el sector principalmente a partir del año 2013, se ha visto enfocada deliberadamente a la prestación de servicios de postventa y mantenimiento automotriz, como medidas reactiva a la disminución de las ventas y la baja en resultados netos, que las empresas aspiran alcanzar en los siguientes años.

La Zona Centro del Ecuador es la más extensa del país con un área de 44.899 km2, está conformada por las provincias de Chimborazo, Cotopaxi, Pastaza y Tungurahua y es considerada como una de las zonas con mayor influencia luego de Quito y Guayaquil en el comercio nacional, dentro de ello sobresale las influencia del sector automotriz como uno de los pilares clave de la economía local. Según datos de la AEADE (2014), la provincia de Tungurahua representaba para el año 2014 el 6,86\% de participación de ventas del Ecuador y las provincias de Chimborazo, Cotopaxi, Pastaza representaban el 8,47\%, es decir un total de 15,33\% de participación total de la Zona Centro para ese año.

En este contexto, se torna relevante estudiar los diferentes enfoques estratégicos adoptados por las empresas del sector automotriz de la zona centro del Ecuador y la manera en que las estrategias adoptadas fueron determinantes en la mejora de sus niveles de rentabilidad durante los años 2013 al 2015. Mintzberg menciona que por encima de todo, la estrategia afecta el bienestar general de la organización a la vez que establece su rumbo, de modo que esta pueda navegar con coherencia a través de su ambiente, por tanto, la manera en que estas organizaciones se adaptan a su entorno contribuirá a la sostenibilidad de sus resultados en el tiempo

\section{ESTADO DEL ARTE}

Para Calderón, Álvarez y Naranjo (2010), el estudio de la estrategia empieza a vislumbrarse en los trabajos iniciales de Selznick (1957), quien estableció que el esfuerzo gerencial debería centrarse en las fortalezas internas y en la capacidad de gestión para poder generar ventajas frente a otras organizaciones. Pero es con Chandler (1962), Ansoff (1965) y Andrews (1971) que el concepto de estrategia toma cuerpo; el primero de ellos la consideró la base para determinar las metas y los objetivos de una empresa a largo plazo y para la adopción de recursos necesarios para llevar a cabo dichos objetivos; Ansoff (1965), por su parte, la cataloga el hilo conductor entre la empresa y las actividades que generan productos, mientras Andrews (1971) la describe como el patrón de políticas, planes y metas para la consecución de los objetivos, de forma tal que es posible identificar en qué lugar se encuentra la empresa y qué tipo de empresa es o va a ser. Además precisó que en relación con la estrategia hay dos elementos interrelacionados, pero claramente diferenciados: la formulación y la ejecución.

La propuesta de contrastación empírica del concepto y tipología de estrategias fue abordada a finales de la década de los setenta, principalmente con el aporte de Miles y Snow (1978), Porter (1980) y posteriormente una propuesta crítica de Mintzberg (1991). Calderón et al. (2010), señala que Porter $(1980,1985,1996)$ desarrolló un modelo de

ENI AC Projetos, Guarulhos (SP),V.5, n.2, jun.- dez. 2016 
estrategias genéricas y un marco de tipologías en el que establece estrategias genéricas (liderazgo en costo, diferenciación y segmentación) que pueden ser usadas para ubicar la empresa en una industria en particular $y$, por consiguiente, construir una ventaja competitiva.

Porter introdujo el concepto de estrategias genéricas a fin de representar las posiciones estratégicas alternativas en un sector, en su criterio las estrategias genéricas siguen siendo útiles para caracterizar las posiciones estratégicas al nivel más simple y más amplio en las organizaciones. Para Calderón et al. (2010), algunos investigadores han cuestionado las estrategias genéricas porque constituyen un modelo inferior a las dimensiones de la estrategia competitiva de la empresa (Hill, 1988; Wright, 1987), porque no describen la estrategia de forma adecuada (Chrisman et al., 1988; Wright,1987) o porque no presentan claridad en un entorno competitivo global y de cambio tecnológico (Mintzberg, 1988).

La tipología propuesta por Miles y Snow, también ha logrado gran incidencia en la literatura estratégica al haber sido aplicada de manera directa a la explicación del comportamiento de un gran número de organizaciones de diferentes sectores de actividad, el grado de proactividad de la empresa a la hora de adoptar decisiones da lugar a cuatro arquetipos estratégicos: prospectivos, defensores, analizadores y los reactivos. (Cabello, García, Jiménez y Ruiz. 2000).

Los prospectivos son organizaciones enfocadas en la investigación de oportunidades de mercado y en innovar respuestas a tendencias ambientales emergentes; por ello, son frecuentemente los creadores de cambio e incertidumbre en la industria, a costa de no ser totalmente eficientes. Los defensores son organizaciones concentradas en la mejora de la eficiencia de sus operaciones, que rehúyen investigar oportunidades fuera de su estrecho ámbito producto-mercado, y que rara vez necesitan efectuar grandes ajustes en su tecnología, estructura o métodos. Los analizadores son organizaciones que combinan los perfiles de los dos arquetipos anteriores, cada uno de ellos en diferentes negocios; en sus áreas estables, operan rutinaria y eficientemente usando estructuras y procesos formalizados. Por último, los reactivos son organizaciones en las cuales los directivos no perciben el cambio y la incertidumbre ambientales de modo adecuado, por lo que son incapaces de responder efectivamente; estas empresas suelen carecer de una adaptación consistente entre la estrategia y la estructura, e incluso de una estrategia explícita. (Zorsona et al., 2007)

Según Robinson y Perarse (1988), numerosas investigaciones desarrolladas hasta los años 80 en torno a la estrategia y sus resultados, han predicho relaciones lineales entre cuota de mercado, volumen de producción acumulada y ROI. Aunque otros estudios posteriores (Woo, 1983) evidenciaron numerosas excepciones a la regla, Porter (1980) ha asumido la hipótesis de que las empresas que compiten con estrategias de liderazgo en costes tendrán ROI superiores al promedio de la industria con altas cuotas de mercado.

Con relación al desempeño organizacional en torno a la estrategia, Pérez H. (2010), describe en su tesis doctoral que, un gran número de organizaciones han modificado sus sistemas de medición del desempeño (Performance Measurement Systems, PMS), pasando 
a adoptar sistemas estratégicos de medición de desempeño (SPMS) tales como Balanced Scorecards (BSC), Primas de desempeño o nuevas generaciones de tableros de mando (Speckbacher et. al., 2003; Neely, 2007, 2008; Rigby, 2009; Micheli y Manzoni, 2010). No obstante, la literatura sobre gestión estratégica y control de gestión sugiere que no existe una evidencia clara de que una estructura definida de sistema de medición de desempeño afecte al desempeño de las organizaciones. Esto sugiere la posibilidad de que existan otras variables que influyan mediando o moderando dicha relación. El estudio realizado plantea la propuesta de analizar la influencia de variables tales como las decisiones directivas, la intensidad del uso de los sistemas de medición del desempeño y la incertidumbre del entorno, con tal de entender mejor la relación entre los sistemas de medición del desempeño.

\section{METODOLOGÍA}

La investigación contempla un diseño longitudinal y retrospectivo ya que refiere al estudio de siete empresas comerciales e industriales representativas del sector automotriz de la zona centro del Ecuador durante los año 2013 al 2015. Se desarrolló una investigación de campo para recabar información de carácter primaria a través de una encuesta tipo Likert realizada a informantes calificados de las empresas en estudio, que incluyeron 24 preguntas enfocadas a cuatro factores de evaluación: mercado, eficiencia, investigación y estructura; en torno a los cuales se caracterizaron la tipología de estrategias genéricas que adoptaron las empresas, sean estas Prospectiva, Defensiva, Analizadora o Reactiva.

Se evaluó la información de carácter secundaria, relacionada con los Estados Financieros oficiales de las empresas durante el período de estudio, disponibles en las bases de datos oficiales de la Superintendencia de Compañías del Ecuador, los cuales fueron sometidos a un análisis cuantitativo a través de indicadores financieros: ROI (rentabilidad sobre los activos) y EBITDA (Utilidad antes de intereses, impuestos, depreciación y amortización) para medir la variable rentabilidad.

La evaluación del comportamiento de las dos variables nos permite plantear dos enfoques de la investigación, el enfoque cualitativo al medir la variable estrategia organizacional en torno a la identificación de la tipología de estrategia que adoptaron estas empresas y un enfoque cuantitativo al estudiar su rentabilidad comparativamente con los resultados del sector. Para la comprobación de hipótesis se realizó un análisis descriptivo para evaluar la relación existente entre el tipo de estrategia evidenciada y la rentabilidad que presentaron las empresas estudiadas.

\section{RESULTADOS}

\subsection{Estrategia Organizacional}

ENIAC Projetos, Guarulhos (SP),V.5, n.2, jun.- dez. 2016 
Según se muestra en la tabla 1, la estrategia Analizadora es el arquetipo que caracteriza a cinco de las siete empresas estudiadas; de manera general estas empresas ofrecen productos competitivos en el mercado con el fin de evitar riesgos futuros, siendo flexibles a cambios del entorno pero manteniendo políticas de disminución y control de costos y gastos, esto con relación a los factores de mercado y eficiencia. En cuanto a su enfoque a la innovación, para las empresas comerciales identificadas no está a su alcance cambios funcionales de los productos que ofrecen, sin embargo, se orientan con mayor énfasis a la mejora en los servicios de postventa que prestan.

Tabla 1. Tipología de Estrategia adoptadas por las empresas en estudio.

\begin{tabular}{lll}
\hline \multicolumn{1}{c}{ Empresas } & Tipo de empresas & Tipo de Estrategia \\
\hline Automotores de la Sierra (ASSA) & Comercial & Prospectiva \\
Ambacar S.A. & Comercial & Analizadora \\
Automekano S.A. & Comercial & Analizadora \\
Autosierra S.A. & Comercial & Analizadora \\
Ciauto S.A. & Industrial & Analizadora \\
Vehysa S.A. & Comercial & Analizadora \\
Reencauchadora S.A. & Industrial & Defensiva \\
\hline
\end{tabular}

Fuente: Investigación de campo

Elaborado por los autores

Dentro de este grupo de empresas Analizadoras, se identifica una de carácter industrial considerada como la primera ensambladora de autos en la zona central del Ecuador, recientemente constituida.

Los analizadores son organizaciones que evitan riesgos excesivos pero sobresalen en la entrega de productos o servicios enfocándose en procesos de mejora de la calidad, tienen una gama limitada de productos y tecnología, para este tipo organizaciones son inminentes los problemas relacionados con mantener su participación de mercado o buscar nuevas oportunidades, debe mantener monitoreado permanentemente sus niveles de eficiencia de modo que les permita ser los suficientemente flexibles para enfrentar a cambios en el entorno.

Por otro lado, se identificó a una de las siete empresas estudiadas, dentro del arquetipo de Prospectiva, con evidencias de mayor desarrollo con relación a los factores estudiados: mercado, eficiencia, innovación y estructura. Esta empresa durante el periodo en estudio, ha desarrollado sus procesos con orientación a nuevos mercados, abriendo un amplio portafolio de servicios en torno al proceso comercial principal que realiza, tales como financiamiento directo, recepción de autos usados, servicios de postventa, accesorios automotrices, seguros, entre otros. Las actividades de investigación y desarrollo de esta empresa se ha traducido en el diseño de un software especializado para las particularidades del a organización y del negocio automotriz.

En relación a su estructura es una organizativa descentralizada y flexible, por su amplia cobertura geográfica, ha diseñado esquemas de gestión por áreas de especialización

ENI AC Projetos, Guarulhos (SP),V.5, n.2, jun.- dez. 2016 
y mantiene comités directivos enfocados a sus procesos de mejora continua. Esta empresa por su experiencia en el mercado y por el respaldo de la marca Chevrolet, la cual tiene mayor participación en el mercado ecuatoriano, muestra evidencias de un nivel de madurez dentro del ciclo de vida organizacional, acompañado de una estructura financiera robusta y de una imagen muy reconocida en la localidad.

Los Prospectivos, promueven con frecuencia la creatividad por sobre la eficiencia, esto hace que deban concentrar gran cantidad de recursos en actividades que generarán beneficios económicos futuros, por tanto, deberán responder con sólidas estructuras financieras, además, sus procesos de descentralización animan la colaboración de diversos departamentos por lo cual puede verse afectado sus objetivos de especialización.

En la tabla 1, se muestra además que una de las siete empresas estudiadas está considerada dentro de la tipología de Defensiva, esta organización de carácter industrial enfrenta sus acciones hacia un mercado maduro del sector aunque con enfoque diferenciador, ha orientado sus acciones a la mejora de sus procesos productivos, fuertes mecanismos de control y continuidad. Su actividad principal se oriente al servicio de reencauche, apalancado en materia prima de su proveedor mexicano con más de 70 años de experiencia.

Las organizaciones Defensivas tienden a funcionar lo mejor posible en ambientes estables y a largo plazo, por tanto se deberán enfrentar con frecuencia a los cambios del entorno, que hacen que sus procesos técnicos establecidos y estandarizados sean ajustados con frecuencia y deban recurrir al apoyo tecnológico, así mismo deberán buscar la forma de asegurar sus niveles de eficiencia.

Finalmente, no se muestra evidencia que dentro de las empresas estudiadas se identifique un arquetipo Reactiva, es decir aquellas empresas que tienen poco control sobre su ambiente externo y el mercado, carecen de eficientes mecanismos internos de control, de una estrategia y una estructura sistemática.

\subsection{Rentabilidad: ROI y EBITDA}

Los resultados de rentabilidad en las empresas en estudio durante los años 2013 al 2015, medidos a través de la Rentabilidad sobre la Inversión (ROI) y en función de las ganancias o la utilidad obtenida por una empresa, sin tomar en cuenta los gastos financieros, impuestos y depreciación o amortización (EBITDA), se muestran en la Tabla 2. Con relación al ROI, ASSA, Ciauto y Ambacar presentan resultados promedio del período del 6,2\%, 5,6\% y 5,4\% respectivamente, porcentajes inferiores al reportado para esta industria según cifras oficiales de la Superintendencia de Compañías para el año 2015

del $9 \%$ en promedio. Mientras que las cuatro empresas restantes presentan una rentabilidad sobre su inversión, inferior al $4 \%$. 
Tabla 2. Rentabilidad medida a través de ROI y EBITDA

\begin{tabular}{lrrrcrcrrr}
\hline \multirow{1}{*}{ Empresas } & \multicolumn{4}{c}{ ROI } & \multicolumn{5}{c}{ EBITDA } \\
\cline { 2 - 10 } & $\mathbf{2 0 1 3}$ & $\mathbf{2 0 1 4}$ & $\mathbf{2 0 1 5}$ & Promedio & $\mathbf{2 0 1 3}$ & $\mathbf{2 0 1 4}$ & $\mathbf{2 0 1 5}$ & Promedio \\
\hline ASSA & $6,8 \%$ & $5,9 \%$ & $5,9 \%$ & $6,2 \%$ & $\$ 6.771 .085$ & $\$ 1.329 .403$ & $-\$ 86.297$ & $\$ 2.671 .397$ \\
Ambacar S.A. & $4,9 \%$ & $6,4 \%$ & $4,9 \%$ & $5,4 \%$ & $\$ 3.938 .915$ & $\$ 2.653 .828$ & $\$ 2.438 .390$ & $\$ 3.010 .378$ \\
Automekano S.A. & $-1,0 \%$ & $1,1 \%$ & $0,8 \%$ & $0,3 \%$ & $-\$ 1.662 .900$ & $-\$ 84.627$ & $\$ 151.818$ & $-\$ 531.903$ \\
Autosierra S.A. & $1,9 \%$ & $2,8 \%$ & $2,4 \%$ & $2,4 \%$ & $\$ 182.822$ & $\$ 216.840$ & $\$ 173.847$ & $\$ 191.170$ \\
Ciauto S.A. & $9,1 \%$ & $6,3 \%$ & $1,4 \%$ & $5,6 \%$ & $\$ 875.682$ & $\$ 3.310 .620$ & $\$ 1.012 .386$ & $\$ 1.732 .896$ \\
Vehysa S.A. & $4, \%$ & $3,3 \%$ & $4,0 \%$ & $3,8 \%$ & $\$ 612.604$ & $\$ 191.524$ & $\$ 163.992$ & $\$ 322.707$ \\
Reencauchadora S.A. & $-0,6 \%$ & $0,3 \%$ & $3,5 \%$ & $1,1 \%$ & $\$ 1.075 .085$ & $\$ 154.855$ & $\$ 312.299$ & $\$ 514.080$ \\
\hline
\end{tabular}

Fuente: Investigación de campo

Elaborado por los autores

El EBITDA de las empresas durante el período estudiado, muestra comportamientos promedio positivos, excepto para Automekano, que durante los años 2013 y 2014 reportó un desempeño negativo de este indicador, evidenciando la pérdida y resultados marginales alcanzados. Por otro lado, Ambacar presenta en promedio el performance más destacado llegando a más de tres millones de dólares, seguido de ASSA con dos millones y Ciauto con más de un millón de dólares. Este indicador nos permite conocer cuánto puede generar la organización, luego de haber deducido los gastos financieros y tributarios, por tanto si es positivo da muestras del valor que está agregando a la organización los resultados alcanzados.

\subsection{Tipología de Estrategia y Rentabilidad}

Los resultados comparativos de carácter cualitativo y cuantitativo que se muestran en la Tabla 3, nos permite evidenciar que la empresa con los mayores niveles de rentabilidad sobre la inversión (6,2\% promedio) y con resultados positivos de EBITDA (US\$ 2,6 millones), es identificada dentro de la tipología de Prospectiva, comparativamente es la que más cercana se encuentra a los resultados de la industria ( $9 \%$ promedio), por otro lado, la empresa que alcanza el 1,1\% de rentabilidad sobre la inversión y un EBITDA de US\$514.080 es identificada como Defensiva, los resultados marginales de rentabilidad alcanzados para el año 2015 permiten mejorar el desempeño de los años anteriores.

Tabla 3. Tipología de empresas y rentabilidad 


\begin{tabular}{lccr}
\hline \multicolumn{1}{c}{ Empresas } & $\begin{array}{c}\text { Tipología } \\
\text { identificada }\end{array}$ & $\begin{array}{c}\text { ROI } \\
\text { Promedio }\end{array}$ & $\begin{array}{c}\text { EBITDA } \\
\text { Promedio }\end{array}$ \\
\hline ASSA & Prospectiva & $6,2 \%$ & $\$ 2.671 .397$ \\
Ambacar S.A. & Analizadora & $5,4 \%$ & $\$ 3.010 .378$ \\
Automekano S.A. & Analizadora & $0,3 \%$ & $-\$ 531.903$ \\
Autosierra S.A. & Analizadora & $2,4 \%$ & $\$ 191.170$ \\
Ciauto S.A. & Analizadora & $5,6 \%$ & $\$ 1.732 .896$ \\
Vehysa S.A. & Analizadora & $3,8 \%$ & $\$ 322.707$ \\
Reencauchadora S.A. & Defensiva & $1,1 \%$ & $\$ 514.080$ \\
\hline
\end{tabular}

Fuente: Investigación de campo

Elaborado por los autores

El grupo de empresas identificadas como Analizadoras, presentan niveles de Rentabilidad sobre la Inversión que van desde el 2,4\% al 5,6\% promedio, mientras que el EBITDA para estas empresas es positivo durante el período estudiado, exceptuando los resultados de la empresa Automekano que mantiene un comportamiento distinto a las empresas de su grupo.

Según Robinson y Perarse (1988), las investigaciones desarrolladas en torno a la estrategia y sus resultados han predicho relaciones lineales entre cuota de mercado, volumen de producción acumulada y ROI, en este sentido Porter (1980) ha asumido la hipótesis de que las empresas que compiten con estrategias de liderazgo en costes y aquellas que son más estructuradas, tendrán ROI superiores al promedio de la industria con altas cuotas de mercado.

\section{CONCLUSIONES Y RECOMENDACIONES}

Las empresas estudiadas representativas del sector automotriz de la Zona Centro del Ecuador, durante el período correspondiente a los años 2013 al 2015, evidencian en mayor grado características que permiten identificarlas como Analizadoras; dentro de la tipología estratégica propuesta por Miles y Snow y considerando cuatro factores de evaluación: mercado, eficiencia, investigación y estructura. Se identifica además una empresa en condiciones que la definen como Prospectiva y otra como Defensiva.

La rentabilidad de las empresas medida a través de indicadores financieros, muestran con relación al ROI resultados inferiores a los alcanzados por las empresas del sector según datos oficiales, mientras que con relación al desempeño del EBITDA presentan resultados positivos excepto una de ellas.

La relación de paradigmas de comportamiento entre la estrategia y la rentabilidad nos lleva a determinar que la estrategia prospectiva y analizadora identificada en la mayoría

ENI AC Projetos, Guarulhos (SP),V.5, n.2, jun.- dez. 2016 
de empresas de la Zona Centro en el periodo 2013 al 2015 les permitió generar mayores niveles marginales de rentabilidad que aquella que habría aplicado una estrategia de carácter Defensiva, con desempeño comparativamente menor en ROI y EBITDA.

Dada la relación reconocida entre la estrategia adoptada por las organizaciones y los efectos sobre su desempeño, es necesario evaluar que las organizaciones pueden adoptar diferentes respuestas ante entornos parecidos, esta premisa de la elección estratégica es pues que la dirección dispone de la capacidad para elegir entre varias formas organizativas estables, queriendo con ello decir que si una empresa escoge cierta estrategia y diseña una organización coherente con ella, dicha organización será un competidor efectivo durante largo tiempo en la industria. ( Zorsona et al., 2007) El enfoque propuesto por Miles y Snow podría ser evaluado bajo un concepto de ciclo adaptativo en las organizaciones.

\section{REFERENCIAS}

Anuario de la Asociación de Empresas Automotriz del Ecuador. (2014).

Briola, M. (2010). Estrategia organizacional. Universidad de Buenos Aires.

Cabello, C., García, M., Jiménez, A., Ruiz, José. (2000). Tipología estratégica de Miles y Snow y factores competitivos: un análisis empírico. Cuadernos de Economía y Dirección de la empresa, ISSN 1138-5758, Nº 7, 2000, págs. 365-382.

Calderón, G., Álvarez, C.M., \& Naranjo, J.C. (2010). Estrategia competitiva y desempeño organizacional en empresas industriales colombianas. Innovar, 20(38), 13-26.

Camisón, C. Garrigós, F. Palacios, D. (2007). Estrategias competitivas y desempeño empresarial: Estudio comparativo de los modelos de Robinson \& Pearce y Miles \& Snow en el Sector Hotelero Español. Revista Investigaciones Europeas de Dirección y Economía de la Empresa. Vol. 13, No 3, 2007, pp. 161-182, ISSN: 1135-2523.

Dess, G. \& Davis, P. (1984). Porter's (1980) Genericstrategies as determinants of strategicgroupmembership and organizational performance. Academy of Management Journal, 27(3), 467-488.

Miles, R.E. y Snow, (1978): Organizacional Strategy, Structure and Process, McGraw-Hill, Nueva York.

Mintzbert, H. (1983). Diseño de Organizaciones eficientes. Editorial Prentice Hall. Reimpresión Argentina (1991).

Mintzbert, H. (1991). Diseño de Organizaciones eficientes. Argentina. Editorial El Ateneo.

ENI AC Projetos, Guarulhos (SP),V.5, n.2, jun.- dez. 2016 
Mintzbert, H. (1991). Mintzbert y la Dirección. España. Editorial Diaz de Santos S.A.

Pérez, H. (2010). Tesis Doctoral. La influencia de los sistemas estratégicos de medición de desempeño sobre las decisiones empresariales. Universidad Ramon Llull. Barcelona España.

Porter, M. (1980). CompetitiveStrategy, Free Press, New York, versión en castellano (1982): Estrategia Competitiva. Técnicas para el Análisis de los Sectores Industriales y de la Competencia, CECSA, México.

Porter, M. (1999). Ser competitivo: nuevas aportaciones y conclusiones.Bilbao: Ediciones Deusto.

Porter, M. (2011). Harvard Business Review. Diciembre 2011.

Robinson, R. B. y Pearce, J.A. (1988): "Plannedpatterns of strategicbehavior and theirrelationship tobusiness-unit performance”. Strategic Management Journal, N. 9, pp. 43-60.Rumelt, R.P., Schendel, D. y Teece, D.J. (1991): "Strategicmanagement and economics”. StrategicManagement Journal, Vol. 12, pp. 5-29.

Sheppeck, M. \&Militello, J. (2000). Strategic HR configurations and organizacional performance. Human Resource Management, 39(1), 5-16. 